\title{
Mannheim Carotid Intima-Media Thickness Consensus (2004-2006)
}

\author{
An Update on Behalf of the Advisory Board of the 3rd and 4th Watching the Risk \\ Symposium 13th and 15th European Stroke Conferences, Mannheim, Germany, 2004, and \\ Brussels, Belgium, 2006
}

\author{
P.J. Touboul M.G. Hennerici S. Meairs H. Adams P. Amarenco N. Bornstein L. Csiba \\ M. Desvarieux S. Ebrahim M. Fatar R. Hernandez Hernandez M. Jaff S. Kownator \\ P. Prati T. Rundek M.Sitzer U.Schminke J.-C. Tardif A. Taylor E. Vicaut K.S. Woo \\ F. Zannad M. Zureik
}

\section{Key Words}

Intima-media thickness - Consensus - Vascular ultrasound • Randomized clinical trials $\cdot$ Carotid diseases

\begin{abstract}
Intima-media thickness (IMT) is increasingly used as a surrogate end point of vascular outcomes in clinical trials aimed at determining the success of interventions that lower risk factors for atherosclerosis and associated diseases (stroke, myocardial infarction and peripheral artery diseases). The necessity to promote further criteria to distinguish early atherosclerotic plaque formation from thickening of IMT and to standardize IMT measurements is expressed through this updated consensus. Plaque is defined as a focal structure that encroaches into the arterial lumen of at least $0.5 \mathrm{~mm}$ or $50 \%$ of the surrounding IMT value or demonstrates a thickness $>1.5 \mathrm{~mm}$ as measured from the media-adventitia interface to the intima-lumen interface. Standard use of IMT measurements is based on physics, technical and disease-related principles as well as agreements on how to perform, interpret and document study results. Harmonization of carotid image acquisition and analysis is needed for the comparison of the IMT results obtained from epidemiological and interventional studies around the world. The consensus con-
\end{abstract}

cludes that there is no need to 'treat IMT values' nor to monitor IMT values in individual patients apart from exceptions named, which emphasize that inside randomized clinical trials should be performed. Although IMT has been suggested to represent an important risk marker, according to the current evidence it does not fulfill the characteristics of an accepted risk factor. Standardized methods recommended in this consensus statement will foster homogenous data collection and analysis. This will help to improve the power of randomized clinical trials incorporating IMT measurements and to facilitate the merging of large databases for metaanalyses.

Copyright $\odot 2007$ S. Karger AG, Basel

\section{Introduction}

Decades of silent arterial wall alterations precede vascular clinical events, which then reflect advanced atherosclerotic disease. The first morphological abnormalities of arterial walls can be visualized by B-mode ultrasonography. This high-resolution, noninvasive technique is one of the best methods for the detection of early stages of atherosclerotic disease, because it is rapidly applicable, readily available and demonstrates the wall structure

\section{KARGER}

Fax +41613061234 E-Mail karger@karger.ch www.karger.com (c) 2007 S. Karger AG, Basel

1015-9770/07/0231-0075\$23.50/0

Accessible online at:

www.karger.com/ced
Dr. Pierre-Jean Touboul

Hôpital Bichat, Service du Pr. Amarenco

46 Rue Raymond Huchard

FR-75018 Paris (France)

Tel. +3366042 0333, Fax +33 14061 0162, E-Mail pjtw@noos.fr 
with better resolution than any other similar technique (e.g. magnetic resonance imaging). Accordingly, ultrasound has been used in a number of studies to monitor the intima-media thickness (IMT) of the carotid arteries, a measurement which has consequently been shown to be associated with cardiovascular risk factors and the incidence of cardiovascular disease. However, these measurements are not strictly associated with individual risks of stroke, myocardial infarction and peripheral artery disease. Furthermore, there are diverse approaches for measuring IMT, and some of these may lead to divergent results. Moreover, there are no unified criteria for distinguishing atherosclerosis as seen in early plaque formation from thickening of the intimal-medial complex. This is because IMT reflects not only early atherosclerosis, but also nonatherosclerotic compensatory enlargement with largely medial hypertrophy as a result of smooth muscle cell hyperplasia and fibrocellular hypertrophy. This differentiation is important because epidemiological studies have shown that wall thickening as depicted by ultrasonographic measurements of IMT is different from atherosclerotic plaque regarding localization, natural history, risk factors and predictive value for vascular events. As IMT is being increasingly used in clinical trials to serve as a surrogate end point for determining the success of interventions that lower risk factors for atherosclerosis, it is imperative that standardized methods be used to allow homogenous data collection and analysis. This would help to improve the power of such studies and to facilitate the merging of large databases for meta-analyses.

This consensus statement addresses the important issue of standardization of carotid IMT measurements and seeks to clarify problems related to the classification of early atherosclerotic lesions. It is an update of the Mannheim IMT consensus document published in 2004.

\section{IMT and Early Plaque}

In the absence of atherosclerotic plaque, B-mode ultrasound displays the vascular wall as a regular pattern that correlates with anatomical layers. The intima-media portion of this pattern is represented by the area of tissue starting at the luminal edge of the artery and ending at the boundary between the media and the adventitia. This interface is well depicted by ultrasound. With increasing age, this pattern has been shown to thicken in a uniform way in straight arterial segments. Thickening of the intima-media is accelerated and enhanced in the presence of risk factors of atherosclerosis, particularly high blood pressure, and by inherited genetic factors. As a mirror of these processes, IMT was identified as a tool to investigate normal aging and preclinical atherosclerosis. Later stages of atherosclerosis (plaque, stenosis, occlusion) can also be identified by ultrasound imaging either in the absence of or coincident with increasing IMT. However, there are intermediate stages between increased IMT and atherosclerotic plaque formation that cannot clearly be differentiated either by ultrasound or by histological examination. Such conditions are common at the bifurcation and the origin of the internal carotid artery, but occur only occasionally in the common carotid artery (CCA). Epidemiological and intervention studies have shown that although both share some common atherosclerosis risk factors, the natural history, patterns of risk factors and the prediction of cardiac and cerebral events are different for carotid IMT and carotid plaque.

The consensus recommends the following definitions for ultrasound characterization of IMT and atherosclerotic plaque:

(1) IMT is a double-line pattern visualized by echotomography on both walls of the CCAs in a longitudinal image. It is formed by two parallel lines, which consist of the leading edges of two anatomical boundaries: the lumen-intima and media-adventitia interfaces.

(2) Plaque is a focal structure encroaching into the arterial lumen of at least $0.5 \mathrm{~mm}$ or $50 \%$ of the surrounding IMT value, or demonstrates a thickness $>1.5 \mathrm{~mm}$ as measured from the media-adventitia interface to the intimalumen interface.

These definitions will allow classification of the vast majority of carotid lesions observed with ultrasound.

Carotid examination includes visualization of common, internal and external carotid arteries. The different ultrasound devices are continuously evolving, providing higher spatial and density resolutions, and improving information on all the structures visualized is waiting for a scientific validation of the superiority of 3-dimensional over 2-dimensional imaging. We propose some rules to improve 2-dimensional image acquisition based on ultrasound physics and basic anatomy.

Utrasound physics principles:

- Ultrasound beam is a virtual biconcave lens which is thicker on the near and distal part of the field represented on the screen. For that reason, the best resolution is commonly obtained in the mid part of this field.

- Perpendicularity between the ultrasound beam and the structures to be visualized provides the best information as the reflection of the incident beam is optimal. 
- The energy of the ultrasound beam decreases with the distance and is usually reduced in the far part of the field.

- Frequency of emission determines the resolution. The higher the frequency, the higher the resolution, however, frequency is inversely proportional to the depth to explore.

- Linear transducers activate piezo-electric crystals simultaneously resulting in a synchronized propagation of the ultrasound wave. Sector scanners are typically activating crystals sequentially in pre-defined time intervals, causing asymmetric shifts and reception of skewed wave fronts. Linear ultrasound transducers therefore provide best image quality for superficial arteries, while electronic sector scanners are better used for deep structures with less accessibility.

Physiological and pathophysiological principles:

- Common and internal carotid arteries are quite homogeneous in structure and hemodynamics. Delimited at the origin by the brachio-arterial cephalic trunk on the right side and the aortic arch on the left side, the CCA ends at the bifurcation which is represented anatomically by the point of divergence of the walls of the CCA (fig. 1). The bifurcation is rather heterogeneous between individuals. Since atherosclerosis usually starts in the carotid bulb, delineation of the different segments is required.

- The continuous related progression of vascular wall changes best monitored in CCA IMT studies is different from discontinuous focal lesions (plaque) which are characteristic of atherosclerotic disease. Therefore, distinction between IMT and plaque must be clearly specified in the scanning protocols.

\section{Where to Measure?}

The CCA can be assessed in nearly every patient. Successful examination of the internal carotid artery and of the carotid bulb depends both upon the anatomical topography of the patient and on sonographer expertise.

(1) Measurement of IMT is most simply performed in a region free of plaque where the double-line pattern is observed - this is advantageous as measurements are easier, more accurate, reproducible and can be standardized by computerized analyses.

(2) IMT can be measured in the CCA, at the bulb and the origin of the internal carotid artery.

(3) In study designs that include wall thickness, values obtained from different sites of the carotid arteries should be documented separately.

Mannheim Carotid Intima-Media

Thickness Consensus

\section{How to Analyze?}

CCA acquisition with ultrasound should be conducted as follows.

Standard equipment includes a high-resolution Bmode system operating with preferentially linear ultrasound transducers at frequencies $>7 \mathrm{MHz}$. Appropriate depth of focus (e.g. $30-40 \mathrm{~mm}$ ), frame rate optimally $25 \mathrm{~Hz}(>15 \mathrm{~Hz}$ ), and gain settings (minimal intraluminal artifacts) are recommended to obtain optimal image quality. Log gain compensation should be around $60 \mathrm{~dB}$. They must be adjusted to obtain a symmetrical brightness on near and far wall, decreasing if necessary the gain in the mid part of the field to avoid intraluminal artifacts.

Carotid IMT scanning and reading protocol recommendations:

(1) The arterial wall segments should be assessed in a longitudinal view, strictly perpendicular to the ultrasound beam, with both walls clearly visualized in order to achieve diameter measurements. Lateral probe incidence is recommended as it offers the best view in the middle field, where the resolution is known to be greater than in the near or far field. A horizontal display of the arterial image is also recommended to obtain the optimal interfaces between blood and vascular structures, on the longest possible segment. The localization of the end of the CCA is necessary to help in repositioning during the follow-up.

(2) IMT should be measured preferably on the far wall. This is because IMT values from the near wall depend in part on gain settings and are less reliable. If taken on the near wall, IMT should be measured separately from IMT of the far wall.

(3) Along a minimum of $10 \mathrm{~mm}$ length of an arterial segment, a high-quality image acquisition is required for serial reproducible measurements. Due to vessel tortuosity, IMT measurement could only be possible at a shorter vessel segment, especially in the carotid bifurcation or the ICA bulb.

(4) Edge detection systems that are properly calibrated provide accurate measurements of IMT. Observations made by readers may be equally valid, but they require rigorous quality control and quality assurance. In addition, manual and semi-manual reading methods are extremely time consuming in comparison to automated systems, which can provide the mean maximal value of 150 measurements performed on a $10-\mathrm{mm}$ segment of CCA in a very short time $(<0.1 \mathrm{~s})$.

Cerebrovasc Dis 2007;23:75-80 


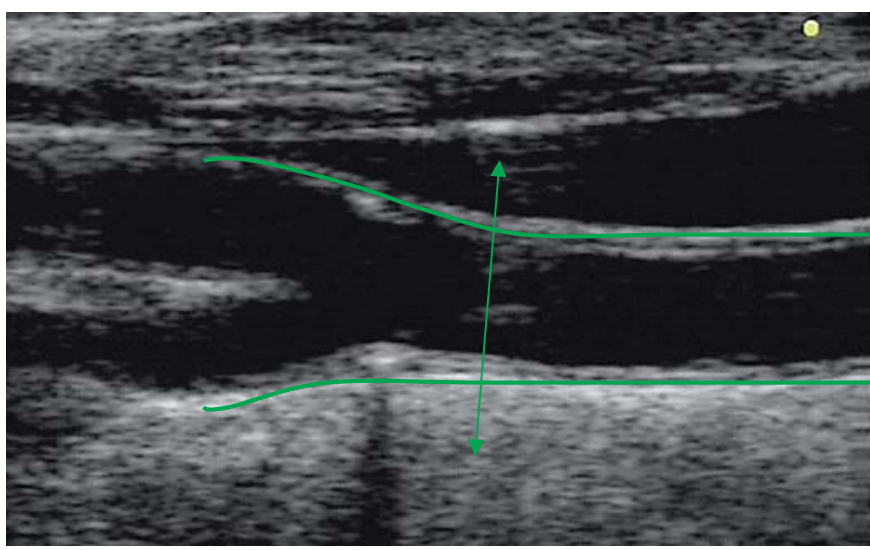

Fig. 1. Longitudinal view of CCA and carotid bifurcation and origin of internal and external carotid arteries. The double arrow line corresponds to the end of the CCA, where near and far walls start diverging.

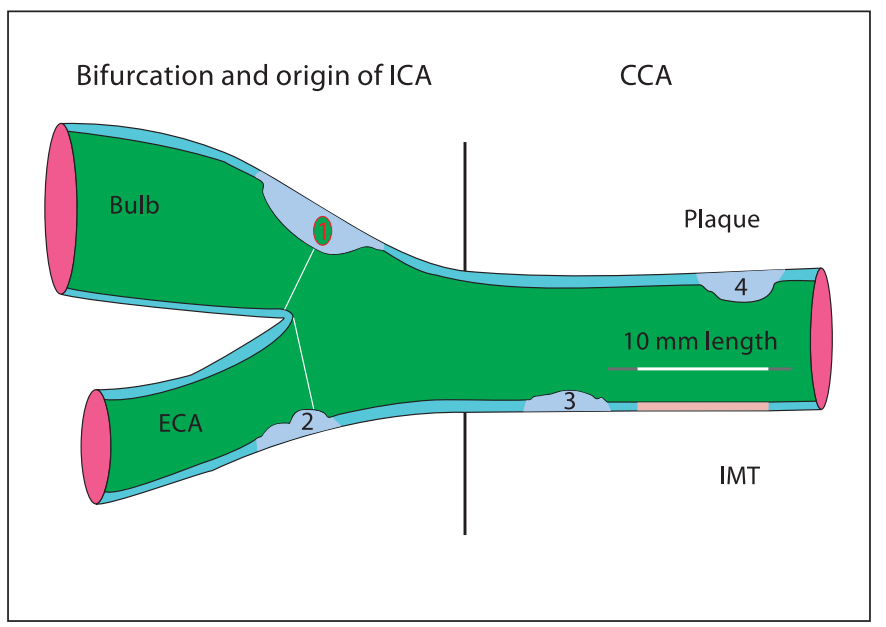

Fig. 3. Drawn representation of carotid tree, with plaque and IMT measurement according to Mannheim consensus. 1: thickness $>1.5 \mathrm{~mm} ; 2$ : lumen encroaching $>0.5 \mathrm{~mm} ; 3,4:>50 \%$ of the surrounding IMT value.

(5) Adventitia-to-adventitia diameter and intraluminal diameter of CCA must also be measured as IMT is significantly correlated with the arterial diameter.

(6) Another important issue is the question which IMT measure (e.g. mean IMT value, maximal IMT value, composite measures from the left and the right side or from different arterial sites) should be used. There is no definite answer to this question. It seems to be plausible that mean IMT values as averaged across the entire dis-

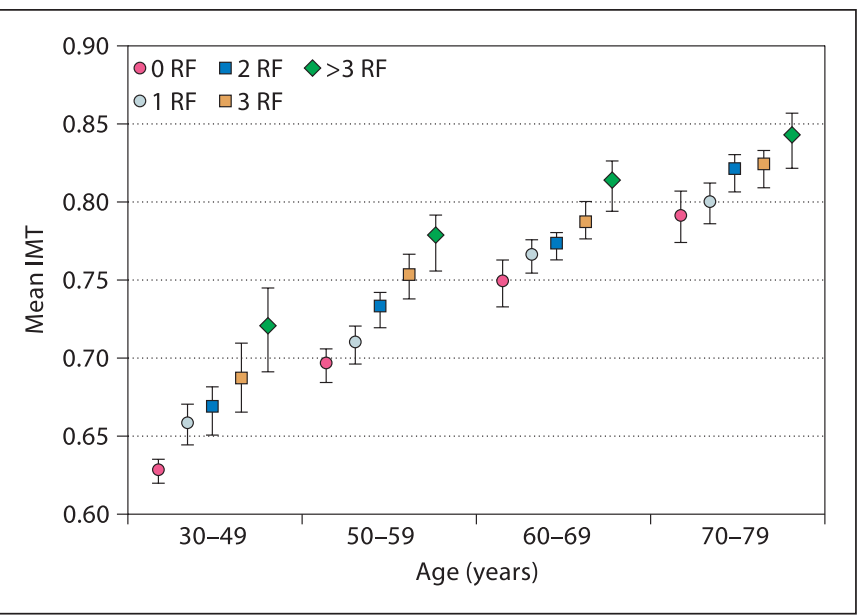

Fig. 2. Age-related quartiles of risk factors (RF). No patients between 30 and 39 years have $>3$ risk factors.

tance are less susceptible to outliers, whereas the maximal IMT may reflect more advanced stages with focal thickening towards plaque formation. It is accepted that IMT values from the left and right side could be averaged although there is a significant difference between the left and right CCA IMT, with higher values on the left side [Foerch et al., 2003]. Composite scores including both plaque and IMT measure should be avoided. In conclusion, data acquisition should be as detailed as possible, and data analysis can be restricted to single measures to reduce multiple testing problems.

(7) Each vascular laboratory must perform periodical quality control of their equipment (phantom scans) and reliability studies of scans and measurements for ultrasonographers and readers. The intraclass correlation coefficient should be evaluated for intra- and interobserver variability, both for IMT and plaque measurements.

\section{Whom to Assess?}

Standard and regular use of IMT measurements are recommended in all epidemiological and interventional trials dealing with vascular diseases to better characterize the population investigated (similarly to the documentation of other risk factors). Determination of 'normal values' should help to better characterize 'intermediate' risk populations in the future (fig. 2). The moderate but significant correlation between IMT and Framingham score observed in a large population (PARC Study) raises the question of the best indicator for vascular event 
in individuals. IMT measurement is the first candidate to evaluate against conventional evaluation of cardiovascular risk. In some countries recommendations already suggest that carefully performed IMT measurement can add incremental information to traditional risk factor assessment (European Society of Cardiology Guidelines).

Ancillary studies in clinical trials should be sufficiently powered to contribute to evaluating the predictive value of IMT on reduction of clinical events.

There is no recommendation up to date to 'treat IMT values' nor to monitor IMT values in individual patients.

\section{Who Should Analyze?}

In clinical trials, several additional requirements are recommended to assure data harmonization. They can be performed through an expert IMT panel as usually represented in the Steering Committees.

Clinical trials using IMT and plaque as intermediate outcomes need to address the following:

(1) study design,

(2) study control,

(3) full image monitoring,

(4) statistical plan analysis,

(5) data monitoring (from CRO and Core Lab),

(6) result analysis

and define:

(1) inclusion criteria for the participating ultrasound centers,

(2) equipment installation if necessary,

(3) sonographers' training and performance control,

(4) certification of the centers (equipment and sonographers),

(5) quality assurance and quality control,

(6) central reading facilities,

(7) evaluation of data variability through intraclass correlation coefficient.

All these procedures can reduce measurement variability, which is a key parameter for a high-quality study, statistical power and sample size determination.

\section{Which End Point to Analyze?}

In studies where clinical outcome parameters are defined, IMT, measured separately if bifurcation and bulb are concerned, and plaque measurements should be included as secondary end points. In studies without clini- cal end points, measurements of IMT and plaque may represent a primary outcome. The optimal choice of primary outcome will depend on study design and primary research hypothesis. IMT and plaque measurements including maximal or mean IMT, plaque thickness, area and volume, and plaque score may all be useful imaging outcomes (fig. 3).

\section{Conclusions}

Recent studies have shown that reduction of IMT values is significantly correlated with risk reduction and improvement of risk factor profiles in large populations. However, neither positive nor negative predictive values on ischemic risk reduction are known in individual subjects treated successfully for specific risk factors. Thus, although IMT has been suggested to represent an important risk marker, it does not fulfill the characteristics of an accepted risk factor. At present, carotid IMT is not an FDA approved surrogate end point of vascular events. The moderate but significant correlation between IMT and Framingham score observed in a large population (PARC Study) raises the question of the best indicator for vascular event in individuals. IMT measurement is the first candidate to evaluate against conventional evaluation of cardiovascular risk in prospective studies. Clinical trials aimed to show that the efficacy of various classes of drugs on the reduction of IMT translates into the reduction of vascular events are needed and are currently underway. 


\section{Reference}

Foerch C, Buehler A, von Kegler S, Sitzer M: Intima-media thickness side differences are limited to the common carotid artery. Hypertension 2003;42:e17, author reply e18.

\section{Additional References}

Ebrahim S, Papacosta O, Whincup P, Wannamethee G, Walker M, Nicolaides AN, Dhanjil S, Griffin M, Belcaro G, Rumley A, Lowe GD: Carotid plaque, intima media thickness, cardiovascular risk factors, and prevalent cardiovascular disease in men and women: The British Regional Heart Study. Stroke 1999;30:841-850.

Glagov S, Weisenberg E, Zarins CK, Stankunavicius R, Kolettis GJ: Compensatory enlargement of human atherosclerotic coronary arteries. N Engl J Med 1987;316:1371-1375.

Hennerici M, Kleophas W, Gries FA: Regression of carotid plaques during low density lipoprotein cholesterol elimination. Stroke 1991; 22:989-992.

Kanters SDJM, Algra A, van Leuween MS, Banga JD: Reproducibility of in vivo carotid intima-media thickness measurements: a review. Stroke 1997;28:665-671.

Lorenz MW, von Kegler S, Steinmetz H, et al: Carotid intima-media thickening indicates a higher vascular risk across a wide age range: prospective data from the Carotid Atherosclerosis Progression Study (CAPS). Stroke 2006;37:87-92.
Luedemann J, Schminke U, Berger K, et al: Association between behavior-dependent cardiovascular risk factors and asymptomatic carotid atherosclerosis in a general population. Stroke 2002;33:2929-2935.

Magyar MT, Szikszai Z, Balla J, Valikovics A, Kappelmayer, J, Imre S, Balla G, Jeney V, Csiba L, Bereczki D: Early-onset carotid atherosclerosis is associated with increased intimamedia thickness and elevated serum levels of inflammatory markers. Stroke 2003;34:5863.

Van der Meer IM, Iglesias del Sol A, Hak AE, Bots ML, Hofman A, Witteman JCM: Risk factors for progression of atherosclerosis measured at multiple sites in the arterial tree: The Rotterdam Study. Stroke 2003;34:23742379.

Pignoli P, Tremoli E, Poli A, Oreste P, Paoletti R: Intimal plus medial thickness of the arterial wall: a direct measurement with ultrasound imaging. Circulation 1986;74:1399-1406.

Rundek T, Elkind MS, Pittman J, et al: Carotid intima-media thickness is associated with allelic variants of stromelysin-1, interleukin6, and hepatic lipase genes: The Northern Manhattan Prospective Cohort Study. Stroke 2002;33:1420-1423.

Steinke W, Els T, Hennerici M: Compensatory carotid artery dilatation in early atherosclerosis. Circulation 1994;89:2578-2581.

Taylor AJ, Burke AP, O’Malley PG, Farb A, Malcom GT, Smialek J, Virmani R: A comparison of the Framingham risk index, coronary artery calcification, and culprit plaque morphology in sudden cardiac death. Circulation 2000;101:1243-1248.
Touboul PJ, Elbaz A, Koller C, Lucas C, Adrai V, Chedru F, Amarenco P: Common carotid artery intima-media thickness and brain infarction: The Etude du Profil Génétique de l'Infarctus Cérébral (GENIC) case-control study. The GENIC Investigators. Circulation 2000;102:313-318.

Touboul PJ, Hennerici M: Epaisseur intima media AVC et traitements médicamenteux 'IMT, Drugs and Stroke'. Paris, Datebe, 2002, p 108.

Touboul PJ, Hennerici MG, Meairs S, Adams H, Amarenco P, Desvarieux M, Ebrahim S, Fatar M, Hernandez Hernandez R, Kownator S, Prati P, Rundek T, Taylor A, Bornstein N, Csiba L, Vicaut E, Woo KS, Zannad F: Mannheim intima-media thickness consensus. On behalf of the advisory board of the 3rd Watching the Risk Symposium 2004, 13th European Stroke Conference, Mannheim, Germany, May 14, 2004. Cerebrovasc Dis 2004; 18:346-349.

Touboul PJ, Prati P, Scarabin PY, Adrai V, Thibout E, Ducimetiere P: Use of monitoring software to improve the measurement of carotid wall thickness by B-mode imaging. J Hypertens Suppl 1992;10:S37-S41.

Touboul PJ, Vicaut E, Labreuche J, et al: Correlation between the Framingham risk score and intima media thickness: The Paroi Arterielle et Risque Cardio-vasculaire (PARC) study. Atherosclerosis , 2006, E-pub ahead of print.

Woo KS, Chook P, Raitakari OT, McQuillan B, Feng JZ, Celermajer DS: Westernization of Chinese adults and increased subclinical atherosclerosis. Arterioscler Thromb Vasc Biol 1999;19:2487-2493. 